\title{
Induced-Charge Electrokinetic Phenomena: Theory and Microfluidic Applications
}

\author{
Martin Z. Bazant* \\ Department of Mathematics and Institute for Soldier Nanotechnologies, Massachusetts Institute of Technology, \\ Cambridge, Massachusetts 02139, USA \\ Todd M. Squires \\ Departments of Applied and Computational Mathematics and Physics, California Institute of Technology, \\ Pasadena, California 91125, USA \\ (Received 17 June 2003; published 10 February 2004)
}

\begin{abstract}
We give a general, physical description of "induced-charge electro-osmosis" (ICEO), the nonlinear electrokinetic slip at a polarizable surface, in the context of some new techniques for microfluidic pumping and mixing. ICEO generalizes "ac electro-osmosis" at microelectrode arrays to various dielectric and conducting structures in weak dc or ac electric fields. The basic effect produces microvortices to enhance mixing in microfluidic devices, while various broken symmetries - controlled potential, irregular shape, nonuniform surface properties, and field gradients - can be exploited to produce streaming flows. Although we emphasize the qualitative picture of ICEO, we also briefly describe the mathematical theory (for thin double layers and weak fields) and apply it to a metal cylinder with a dielectric coating in a suddenly applied dc field.
\end{abstract}

DOI: 10.1103/PhysRevLett.92.066101

The advent of microfluidic technology raises the fundamental question of how to pump and mix fluids at micron scales, where pressure-driven flows and inertial instabilities are suppressed by viscosity [1,2]. The most popular nonmechanical pumping strategy is based on electro-osmosis - the effective slip $\mathbf{u}_{\|}$at a liquidelectrolyte/solid interface due to tangential electric field $\mathbf{E}_{\|}$. The Helmholtz-Smoluchowski formula,

$$
\mathbf{u}_{\|}=-\frac{\varepsilon \zeta}{\eta} \mathbf{E}_{\|}
$$

gives the slip in terms of the permittivity $\varepsilon$ and viscosity $\eta$ of the liquid and the zeta potential $\zeta$ across the diffuse part of the (thin) interfacial double layer [3]. The usual case of constant (possibly nonuniform [4]) $\zeta$, however, has some drawbacks related to linearity, $\mathbf{u}_{\|} \propto \mathbf{E}_{\|}$: (i) the flow is somewhat weak, e.g., $u_{\|}=70 \mu \mathrm{m} / \mathrm{s}$ in aqueous solution with $E_{\|}=100 \mathrm{~V} / \mathrm{cm}$ and $\zeta=10 \mathrm{mV}$ and (ii) ac fields, which reduce undesirable Faradaic reactions, produce zero time-averaged flow.

These drawbacks do not apply to ac electro-osmosis, recently discovered by Ramos et al. [5] and Ajdari [6]. Nonlinear electro-osmotic slip is produced at microelectrodes as an ac field acts on induced double-layer charge prior to complete screening. In spite of extensive work, however, this promising effect remains limited to quasiplanar pairs [7] or arrays [8] of electrodes at a single ac frequency $\omega_{c}=\tau_{c}^{-1}$, where $\tau_{c}=\lambda_{D} L / D$ is the " $R C$ time" of an equivalent circuit of bulk resistors of size $L$ (the electrode spacing) and double-layer capacitors of thickness $\lambda_{D}$ (the Debye screening length), and $D$ is an ionic diffusivity.

How general is this phenomenon? Nonlinear electroosmotic flows have also been observed at dielectric im-
PACS numbers: 82.45.-h, 47.45.Gx, 47.65.+a, 85.90.+h

purities on electrodes with ac forcing [9] and, more suggestively, at dielectric (nonelectrode) microchannel corners in dc fields [10]. Although it is largely unknown (and uncited) in the West, similar flows have also been studied in the Russian literature on polarizable colloids [11], including the effect of such flows on dielectrophoresis [12]. The unifying principle in these diverse effects is that an applied field acts on its own induced diffuse charge, so we suggest the term "induced-charge electroosmosis" (ICEO) to describe it.

In this Letter, we describe more general ICEO flows which may be produced in microfluidic devices. Before we begin, we stress that ICEO dominates electrokinetics at small total zeta potentials (initial + induced), $\zeta \ll$ $2(k T / e) \log \left(L / \lambda_{D}\right)$, where $k T / e \approx 20 \mathrm{mV}$ is the thermal voltage. When this condition is violated, as in highly charged colloids, surface conduction leads to other "nonequilibrium electrosurface phenomena" (NESP) [13], and at very large voltages (above a limiting current) bulk space charge may also drive "second-kind electrokinetic phenomena" [14]. Such exotic effects may be useful in microfluidics [15], but we focus on small voltages, which are often preferable in real devices.

ICEO is nicely illustrated by the flow around an uncharged, ideally polarizable cylinder (e.g., an inert metal wire) of radius $a$ in a suddenly applied, weak, uniform field $\mathbf{E}_{b}$. (The analogous problem for a metal colloidal sphere was introduced long ago by Levich [16]; the double-layer polarization was later analyzed in more detail by Simonov and Shilov [17] and the resulting flow by Gamayunov, Murtsovkin, and Dukhin [18].) As shown in Fig. 1(a), the initial electric field lines are those of a conducting sphere in vacuum, perpendicular to the surface, but the ionic current $\mathbf{J}=\sigma \mathbf{E}$ affects the field. 

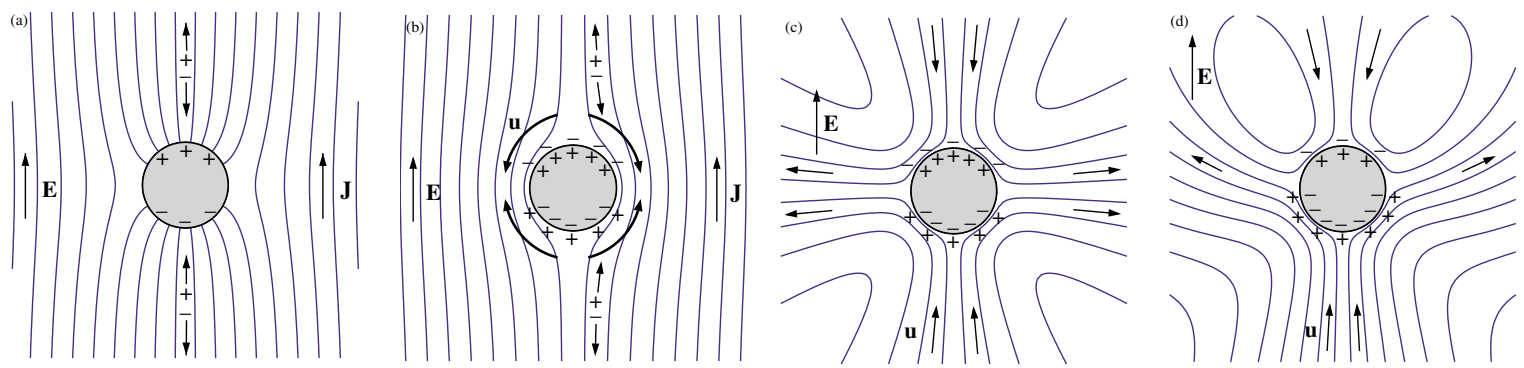

FIG. 1 (color online). Lines of electric field $\mathbf{E}$ (or current $\mathbf{J}=\sigma \mathbf{E}$ ) around a cylindrical metal wire in an electrolyte (a) before and (b) after double-layer charging in response to a suddenly applied dc field and (c) the resulting ICEO streamlines. The flow around a charged polarizable cylinder is shown in $(d)$.

Without surface conduction or Faradaic reactions to transfer the normal current at the surface, ionic charge accumulates in the double-layer "capacitor." As shown in Fig. 1(b), a steady state is reached when the bulk field lines are expelled to become those of an insulator, parallel to the surface. The induced diffuse charge (or $\zeta$ ) shown in Fig. 1(b) is nonuniform-negative $(\zeta>0)$ where the initial current leaves the surface and positive $(\zeta<0)$ where it enters. Since $\mathbf{u}_{\|} \propto-\zeta \mathbf{E}_{\|}$, we can anticipate the quadrupolar ICEO flow shown in Fig. 1(c), which draws in fluid along the field axis and ejects it radially. Reversing $\mathbf{E}$ changes the sign of the induced $\zeta$ everywhere, so the same flow also persists in ac fields (up to the charging frequency $\omega_{c}=D / \lambda_{D} a$ ).

The magnitude of the flow follows easily from dimensional analysis. After charging, the double layer acquires the background voltage across the object (nonuniformly), which produces zeta potentials of order $E_{b} a$ and from Eq. (1) typical flow speeds of order,

$$
U_{0}=\frac{\varepsilon a E_{b}^{2}}{\eta}
$$

(as for a colloidal sphere [18]). When the applied voltage $E_{b} a$ exceeds typical equilibrium zeta potentials $(10 \mathrm{mV})$, ICEO flow exceeds that of standard dc electro-osmosis, e.g., $U_{0}=0.7 \mathrm{~mm} / \mathrm{s}$ in water for $E_{b}=100 \mathrm{~V} / \mathrm{cm}$ and $a=10 \mu \mathrm{m}$. In that case, the maximum frequency $\omega_{c}$ is of order $10 \mathrm{kHz}$ for $\lambda_{D}=10 \mathrm{~nm}$. (For these parameters, we are also justified in neglecting surface conduction $[3,13]$.)

The symmetry of ICEO flow in Fig. 1(c) suggests the microfluidic devices sketched in Fig. 2. For example, a metal post in a transverse ac or dc field produces local time-averaged flow as in Fig. 1(c). Placed at a cross junction with two pairs of corner electrodes [Fig. 2(a)], ICEO draws liquid in along one channel and forces it out the other, and the flow is easily reversed by changing the field direction. An array of posts in a transverse ac field [Fig. 2(c)] produces microvortices to enhance mixing in passing flows. A different design for a T-junction pump [Fig. 2(b)] employs a metal surface coating, which could wrap around in the third dimension to reduce viscous drag by replacing more of the channel wall with sources of ICEO (see also Fig. 4).
In such devices, streaming flows are easily produced by broken symmetries. As the first example, consider a metal cylinder of nonzero total charge. In a dc field, the ICEO flow shown in Fig. 1(d) (and given below) is simply a superposition of the nonlinear quadrupolar flow described above and the linear streaming flow of electrophoresis, which averages to zero for a freely suspended object. In microfluidic devices, however, there is a new possibility: By controlling an object's potential, its induced total charge can be made to vary in phase with the applied field to produce time-averaged ac streaming. For example, a thin metal post at position $x_{0}$ and potential $\phi_{0}$ between two electrodes imposing a linear potential $\phi=$ $-E_{b} x=V x / L$ generates a streaming flow of order

$$
U_{1}=\frac{\varepsilon\left(\phi_{0}+E_{b} x_{0}\right) E_{b}}{\eta}
$$

In the case of the mixer in Fig. 2(c), a post grounded to an electrode, $\phi_{0}=V$, pumps toward the nearest wall with speeds larger than the (superimposed) fixedtotal-charge flow by a geometry-dependent factor,
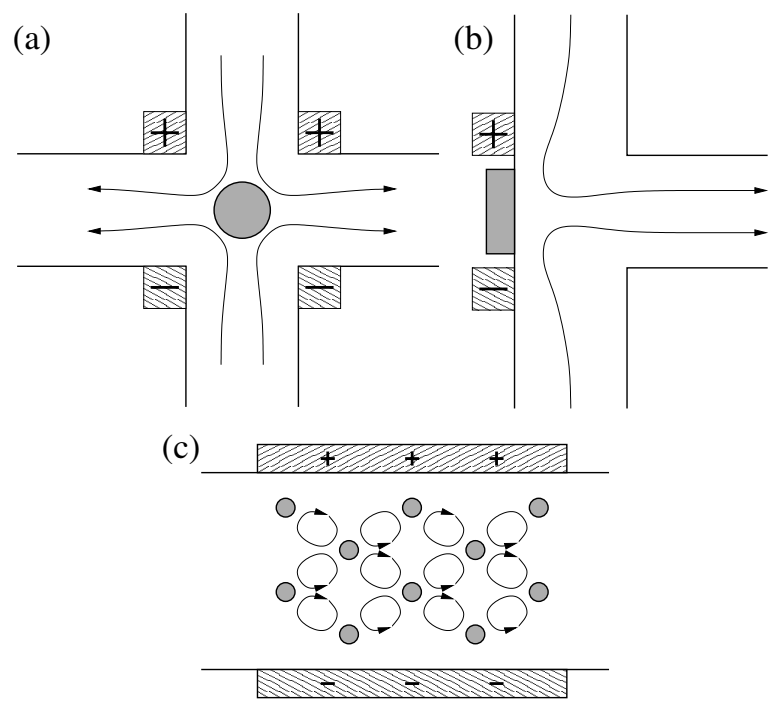

FIG. 2. Simple microfluidic devices exploiting ICEO flows around fixed metal objects (shaded area) driven by small ac voltages at microelectrodes (cross-shaded area). 
$\left|U_{1} / U_{0}\right|=\left(L-x_{0}\right) / a$. Since posts of prescribed potential act as electrodes, this manifestation of ICEO resembles ac electro-osmosis [5,6], but it does not require ac background fields. It is perhaps closer to dc "field-effect flow control" [19], although ICEO involves metal surfaces at much smaller voltages coupled directly to the primary electrodes.

As in the case of ac pumping at electrode arrays $[6,8]$, other broken symmetries also cause a polarizable object to pump fluid via ICEO, if fixed, or move via "inducedcharge electrophoresis" (ICEP), if freely suspended, at the velocity scale $U_{0}$ in either dc or ac fields. For example, consider a metal cylinder with nonuniform surface properties in a uniform field, as in Figs. 3(b) and 3(c). If one side has a greater surface capacitance as in Fig. 3(b), then some induced charge ends up immobilized on the surface unable to cause slip, so the other side "wins" in driving ICEP in its direction. If the sphere is partially insulating as in Fig. 3(c), then primarily the conducting portion is polarized, and the steady-state field acts on the shifted diffuse charge to cause ICEP toward the insulating side.

If the object has an irregular shape as in Fig. 3(d) ICEP occurs in the more protruding direction, where the induced charge is better aligned with the tangential field (as in some experiments [20]). If the same object in Fig. 3(d) were rotated to break left-right symmetry, it would move in the opposite (more rounded) direction, perpendicular to the field axis, albeit unstable to rotations restoring field alignment. Such transverse electro-osmosis has been demonstrated for fixed-charge surfaces in dc fields [4], but with polarizable surfaces it can also occur in ac fields.

The ICEO flows in Fig. 3 motivate the microfluidic devices in Fig. 4, which pump in one direction while mixing via superimposed circulating flows in either ac (a)

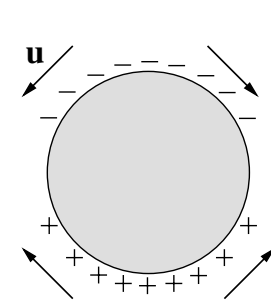

(c)

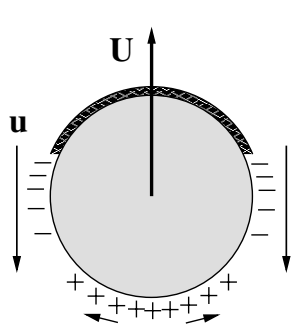

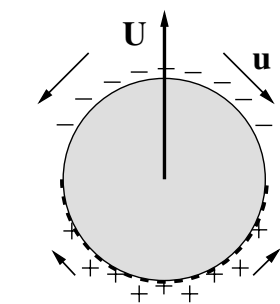

(b)

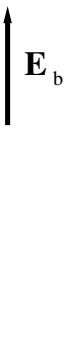

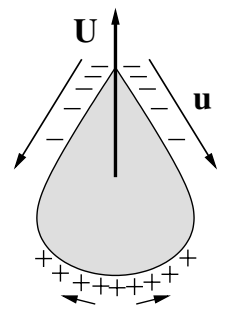

FIG. 3. Sketches of induced diffuse charge $(+,-)$ and ICEO slip $\mathbf{u}$ around (a) a circular cylinder, (b) with a partial coating (dashed line) of increased surface capacitance, (c) with an insulating coating (shaded area), and (d) an asymmetric cross section. In (b)-(d), the ICEP velocity $\mathbf{U}$ points up, regardless of whether the applied field $\mathbf{E}_{b}$ points up or down. or dc fields. For example, asymmetric posts pump and mix in response to an applied field along the channel [Fig. 4(a)], and the pumping direction may be reversed with a transverse field (not shown). An equivalent design [Fig. 4(b)] involves asymmetric grooves in a metallic channel wall. Such features may wrap around the channel in the third dimension, further reducing viscous drag.

Finally, we consider nonuniform applied fields. For example, an uncharged metal post in a nonuniform dc or ac field pumps down the field gradient $\nabla E_{b}$ with a typical streaming velocity

$$
U_{2}=\frac{\varepsilon a^{2}}{\eta}\left|\nabla E_{b}\right|^{2}
$$

which follows from Eq. (1) since induced variations in $\zeta$ are of order $a^{2}\left|\nabla E_{b}\right|$. Such flows profoundly influence dielectrophoresis for polarizable colloidal spheres [12] (via ICEP), but nonspherical shapes require further study. More generally, nonuniform fields provide another useful degree of freedom in ICEO devices. As a simple example, one transverse electrode in the mixer in Fig. 2 could be shortened to produce additional pumping toward the opposite wall (and large-scale circulation) driven by the resulting field gradient. All of this flexibility raises interesting open questions of optimal design.

The physical arguments above can be justified by mathematical analysis of the electrokinetic equations [3] for weak fields and thin double layers, as described in subsequent papers, beginning with Ref. [21]. The origin of ICEO and other NESP is an effective boundary condition on the neutral bulk electrolyte which expresses the conservation of the diffuse charge $q$ in a thin double layer:

$$
-\frac{\partial q}{\partial t}=J_{F}+\hat{n} \cdot \mathbf{J}+\boldsymbol{\nabla}_{s} \cdot \mathbf{J}_{s}
$$

where $J_{F}$ is the Faradaic current, $\hat{n}$ is the outward normal, $\boldsymbol{\nabla}_{s}$ is the surface gradient, and $\mathbf{J}_{s}$ is the surface current. NESP mostly involves steady surface conduction, $\hat{n} \cdot \mathbf{J}=$ $-\boldsymbol{\nabla}_{s} \cdot \mathbf{J}_{s}$, at low ac frequencies, $\omega \ll D / a^{2}$, which

(a)

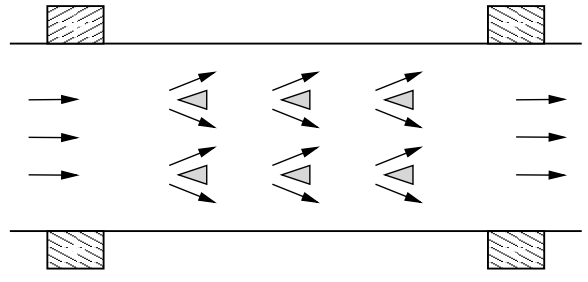

(b)

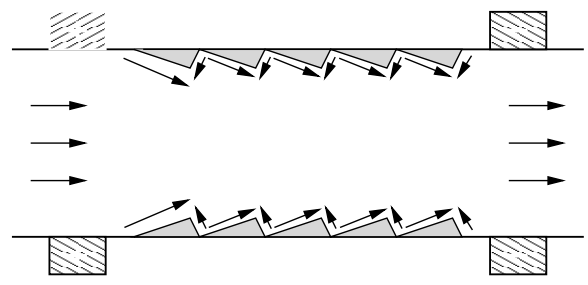

FIG. 4. Microfluidic devices exploiting asymmetric ICEO. 
produces concentration gradients affecting electroosmosis at highly charged surfaces [11,13].

In contrast, ICEO derives from the dominant " $R C$ coupling" for small $\zeta$ (initial + induced) in a homogeneous electrolyte:

$$
C_{D} \frac{\partial \zeta}{\partial t}=\sigma \hat{n} \cdot \mathbf{E}
$$

where $C_{D}(\zeta)=-(d q / d \zeta)$ is the nonlinear differential capacitance per unit area. (Ideal polarizability, $J_{F}=0$, is often a good approximation for small $\zeta$ and ac forcing.) The variable $\zeta$ in Eq. (1) is then obtained by solving Laplace's equation, $\nabla^{2} \phi=0$, subject to the boundary condition (6). For linear screening, $C_{D}=\varepsilon / \lambda_{D}$, such analysis has been done for microelectrode arrays [5-7], while nonlinear screening has also been discussed in colloids [17].

Motivated by the devices in Fig. 2, we calculate the ICEO slip for a metal cylinder at potential $\phi_{0}$ [or $\zeta_{0}=$ $\left.\left(\phi_{0}-\phi_{b}\right) /(1+\delta)\right]$ in a suddenly applied, weak background potential $\phi \sim \phi_{b}-E_{b} r \cos \theta$, where $\zeta=\left(\phi-\phi_{0}\right) /$ $(1+\delta) \ll k T / e$ at $r=a$. (Here $\delta$ is the capacitance ratio of the compact part to the diffuse part of the double layer [6].) The bulk potential,

$$
\phi(r, \theta, t)=\phi_{b}-E_{b} r \cos \theta\left[1+g(t) \frac{a^{2}}{r^{2}}\right],
$$

involves an induced dipole moment, $g(t)=1-2 e^{-t / \tau_{q}}$, effectively changing from a conductor $\left(g=-1, \tau \ll \tau_{q}\right)$ to an insulator $\left(g=1, t \gg \tau_{q}\right)$ at the time scale $\tau_{q}=$ $\tau_{c} /(1+\delta)$. (For nonlinear screening, the poles charge more slowly, $d C_{D} / d|\zeta| \geq 0$, but the steady state is the same.) The slip velocity, $\mathbf{u}_{\|}=u_{\theta} \hat{\theta}$, has two terms:

$$
u_{\theta}(\theta, t)=U_{1}^{\prime} f(t) \sin \theta+2 U_{0}^{\prime} f(t)^{2} \sin 2 \theta,
$$

where $f(t)=1+g(t), \quad U_{1}^{\prime}=\varepsilon\left(\phi_{b}-\phi_{0}\right) E_{b} / \eta(1+\delta)$, and $U_{0}^{\prime}=U_{0} /(1+\delta)$. The first term produces pumping past the cylinder while the second, peaked at $45^{\circ}$ to the field and growing more slowly, produces a symmetric quadrupolar flow. Both terms have nonzero time averages in ac fields, $\left\langle u_{\theta}^{(1)}\right\rangle \propto\left\langle\zeta_{0} E_{b}\right\rangle \sin \theta$ and $\left\langle u_{\theta}^{(0)}\right\rangle \propto a\left\langle E_{b}^{2}\right\rangle \sin 2 \theta$, respectively, although the former requires controlling $\phi_{0}$. Finally, we note that a thin dielectric layer (modeled by $\delta>0$ as in Ref. [6]) reduces ICEO flow by $1 /(1+\delta)$, so care must be taken to keep metal surfaces clean in real devices.

In summary, ICEO is a versatile technique for microfluidic pumping using weak ac (or dc) fields, including many new possibilities other than ac electro-osmosis at planar microelectrode arrays. The remarkable richness of ICEO flows merits further analysis and experiments, especially at moderate voltages where other NESP may become important. Nevertheless, simple drawings, as in Figs. 1-4, suffice for a qualitative understanding in many cases.

This research was supported in part by the U.S. Army through the Institute for Soldier Nanotechnologies, under Contract No. DAAD-19-02-0002 with the U.S. Army Research Office.

*Electronic address: bazant@mit.edu

[1] H. A. Stone and S. Kim, AIChE J. 47, 1250 (2001).

[2] D. J. Beebe, G. A. Mensing, and G. M. Walker, Ann. Rev. Biomed. Eng. 4, 261 (2002).

[3] J. Lyklema, Fundamentals of Interface and Colloid Science (Academic, London, 1991), Vol. 2.

[4] A. Ajdari, Phys. Rev. Lett. 75, 755 (1995); Phys. Rev. E 53, 4996 (1996); 65, 016301 (2001); I. Gitlin et al., Appl. Phys. Lett. 83, 1486 (2003).

[5] A. Ramos et al., J. Phys. D 31, 2338 (1998); J. Colloid Interface Sci. 217, 420 (1999).

[6] A. Ajdari, Phys. Rev. E 61, R45 (2000).

[7] A. González et al., Phys. Rev. E 61, 4019 (2000); N. G. Green et al., Phys. Rev. E 66, 026305 (2002); A. Ramos et al., Phys. Rev. E 67, 056302 (2003).

[8] A. B. D. Brown, C. G. Smith, and A. R. Rennie, Phys. Rev. E 63, 016305 (2001); V. Studer et al., Microelectron. Eng. 61, 915 (2002); M. Mpholo, C. G. Smith, and A. B. D. Brown, Sens. Actuators, B 92, 262 (2003).

[9] F. Nadal et al., Eur. Phys. J. E 9, 387 (2002).

[10] S. K. Thamida and H.-C. Chang, Phys. Fluids 14, 4315 (2002); P. Takhistov, K. Duginova, and H.-C. Chang, J. Colloid Interface Sci. 263, 133 (2003).

[11] V. A. Murtsovkin, Colloid J. 58, 341 (1996), and references therein.

[12] T. S. Simonova, V. N. Shilov, and O. A. Shramko, Colloid J. 63, 108 (2001), and references therein.

[13] S.S. Dukhin, Adv. Colloid Interface Sci. 44, 1 (1993), and references therein.

[14] N. A. Mishchuk and P.V. Takhistov, Colloids Surf. A 95, 119 (1995), and references therein.

[15] Y. Ben and H.-C. Chang, J. Fluid Mech. 461, 229 (2002).

[16] V.G. Levich, Physico-Chemical Hydrodynamics (Prentice-Hall, Englewood Cliffs, NJ, 1962).

[17] I. N. Simonov and V. N. Shilov, Colloid J. USSR 35, 350 (1973); 39, 775 (1977).

[18] N. I. Gamayunov, V. A. Murtsovkin, and A. S. Dukhin, Colloid J. USSR 48, 197 (1986).

[19] R. B. M. Schasfoort et al., Science 286, 942 (1999); K. Ghowsi and R. J. Gale, J. Chromatogr. 559, 95 (1991); S. A. Gajar and M.W. Geis, J. Electrochem. Soc. 139, 2833 (1992).

[20] N. I. Gamayunov and V. A. Murtsovkin, Colloid J. USSR 49, 543 (1987); V. A. Murtsovkin and G. I. Mantrov, Colloid J. USSR 52, 933 (1990).

[21] T. M. Squires and M. Z. Bazant, J. Fluid Mech. (to be published). 